\title{
A Graphical User Interface (GUI) in Matlab to Compute the Thermal Lithospheric Thickness and its Error Bounds
}

\author{
Harini Guruhappa \\ National Geophysical \\ Research Institute \\ (CSIR) India.
}

\author{
Shanti Sree \\ National Geophysical \\ Research Institute \\ (CSIR) India.
}

\author{
C. Madhu \\ National Geophysical \\ Research Institute \\ (CSIR) India.
}

\author{
Kirti Srivastava \\ National Geophysical \\ Research Institute \\ (CSIR) India.
}

\begin{abstract}
Graphical user interface packages in Matlab are getting very popular with the geo-scientific researchers. Matlab GUI (graphical user interface) is a graphical display containing controls which helps in computing and graphically representing the results. In this paper a simple graphical user interface (GUI) viewer is developed in MATLAB that computes the thermal lithospheric structure along with its error bounds. The $\mathrm{m}$-file in the package is integrated through a GUI and the controlling thermal parameters such as crustal thickness, radiogenic heat production, characteristic depth, surface temperature, surface heat flow and thermal conductivity are all given on the screen. The thermal conductivity is considered to be Gaussian random variable with a known coefficient of variability and a correlation length scale. The output is in the form of temperature, depth and its standard deviation. The lithospheric thickness along with the error bounds for the region is then inferred from these graphs. The developed GUI is applied to quantify the lithospheric thickness along with its error structure for any given region where conductive heat transfer is dominant.
\end{abstract}

\section{Key Words}

Graphical User Interface, Matlab, thermal structure, error bounds, Gaussian random variable.

\section{INTRODUCTION}

The subsurface thermal structure of the Earth's crust is influenced by its geothermal parameters such as thermal conductivity, radiogenic heat sources and initial and boundary conditions. Basically two approaches of modeling are commonly used for the estimation of the subsurface temperature field. These are: (1) deterministic approach and (2) the stochastic approach. In the deterministic approach the subsurface temperature field is obtained assuming that the controlling thermal parameters are known with certainty. However, due to inhomogeneous nature of the Earth's interior some amount of uncertainty in the estimation of the geothermal parameters are bound to exist. Uncertainties in these parameters may arise from the inaccuracy of measurements or lack of information about the parameters themselves. Such uncertainties in parameters are incorporated in the stochastic approach and an average picture of the thermal field along with its associated error bounds is determined. The mean value gives the average subsurface thermal picture and the variance or the standard deviation is the variability indicator which gives the errors associated with the thermal structure due to errors in the input controlling parameters.
The uncertainty in the heat flow using a least squares inversion technique incorporating uncertainties in the temperature and thermal conductivities is studied in [1-2]. Later, [3] studied the effect of variation in heat source on the surface heat flow and [4] studied the stochasticity in the thermal models. The small perturbation method is used in [5] to solve the stochastic heat conduction equation with uncertainties in the heat sources to obtain the mean temperature field along with its error bounds. Several researchers have been using the numerical method, the random simulation method to model the error structure in the thermal field incorporating uncertainties in the controlling thermal parameters [6-9]. Stochastic analytical solutions to diffusion problem have been obtained by several researchers. The analytical solution to error bounds on the subsurface temperature depth distribution is obtained by solving the heat conduction equation incorporating Gaussian uncertainties in the thermal conductivity. This analytical solution is coded in Matlab to get numerical solution to the given problem and a GUI has been developed.

Using Matlab programming one can create a GUI which contains controls such as menus, toolbars, buttons and sliders. This can perform any type of computation and display data as plots or as tables. Many Matlab products, such as Curve Fitting Toolbox, Signal Processing Toolbox, and control system Toolbox, include applications with custom user interface [10-11].

A sequence of matlab $\mathrm{m}$ files and two graphical user interfaces to display raw or processed geophysical data to produce the final graphics is developed in [12]. A Matlab based GUI to compute the subsurface thermal structure up to the base of the crust is developed in [13]. Yet another Matlab based GUI is developed in [14] and applied it to understand the hydroseismicity of the Koyna - Warna Region, India.

The analytical expressions for mean and variance in temperature depth distribution have been extended below the crust and closed form solutions obtained. In this paper a simple Matlab GUI (graphical user interface) is developed and the subsurface temperatures computed by giving directly the numerical values of the controlling thermal parameters and the results/plot are displayed instantaneously. The plot of the subsurface thermal field along with its error bounds is computed till we get $1300{ }^{\circ} \mathrm{C}$ mantle adiabat. The depth at which this temperature is reached gives the lithospheric thickness. The study has been applied to Latur earthquake region of India. 


\section{MATHEMATICAL FORMULATION}

The stochastic heat conduction equation with random thermal conductivity is expressed in [15].

$$
\begin{aligned}
\frac{d}{d z}\left\{\left(\bar{K}+K^{\prime}(z)\right) \frac{d T}{d z}\right\} & =-A_{0} e^{-z / D} & & 0 \leq z \leq L \\
& =0 & & z \geq L
\end{aligned}
$$

where $\mathrm{T}$ is the temperature $\left({ }^{\circ} \mathrm{C}\right), \mathrm{A}(\mathrm{z})$ is the radiogenic heat source $\left(\mu W / m^{3}\right), \mathrm{L}$ is the crustal thickness, $\mathrm{D}$ is the characteristic depth, $\mathrm{K}(\mathrm{z})$ is the thermal conductivity $\left(\mathrm{W} / \mathrm{m}^{\circ} \mathrm{C}\right)$ which is expressed as a sum of a deterministic component and a random component where $\overline{\mathrm{K}}$ is the mean value and $\mathrm{K}^{\prime}(\mathrm{z})$ is the random component with mean zero and a Gaussian colored noise correlation structure represented by

$$
\begin{aligned}
& E\left(K^{\prime}(z)\right)=0 \\
& E\left(K^{\prime}\left(z_{1}\right) K^{\prime}\left(z_{2}\right)\right)=\sigma_{K}^{2} e^{-\rho\left|z_{1}-z_{2}\right|}
\end{aligned}
$$

where $\sigma_{\mathrm{K}}^{2}$ is the variance in thermal conductivity, $\rho$ is the correlation decay parameter (or $1 / \rho$ is the correlation length scale) and $z_{1}$ and $z_{2}$ are the depths.

Boundary Conditions are

Constant surface temperature

$$
T=T_{0} \quad \text { at } \quad z=0
$$

$$
\text { Basal heat flow } Q_{B} \quad\left(m W / m^{2}\right)
$$

$$
\bar{K} \frac{d T}{d z}=Q_{B} \quad \text { at } \quad z=L
$$

In [16] the analytical expressions for mean temperatures in the crust is obtained as

$$
\bar{T}=E(T(z))=T_{0}+\frac{Q_{B}}{\bar{K}} z+\frac{A_{0} D^{2}}{\bar{K}}\left(1-\frac{z}{D} e^{-L / D}-e^{-z / D}\right)
$$

The variance in the temperature is obtained as

$$
\begin{aligned}
& \sigma_{T}^{2}=c 1 \times \text { Term } 1+c 2 \times \text { Term } 2+c 3 \times \text { Term } 3+ \\
& c 4 \times \text { Term } 4
\end{aligned}
$$

where $C_{K}=\frac{\sigma_{K}}{\bar{K}}$ is the coefficient of variability in thermal conductivity and

$$
\begin{aligned}
& c 1=C_{K}^{2} A_{0}^{2}(1-\rho D)^{2} / \bar{K}^{2} \\
& c 2=C_{K}^{2} A_{0} \rho(\rho D-1)\left(Q_{B}-A_{0} D e^{-L / D}\right) / \bar{K}^{2} \\
& \text { c3 }=\mathrm{c} 2 \\
& c 4=C_{K}^{2} \rho^{2}\left(Q_{B}-A_{0} D e^{-L / D}\right)^{2} / \bar{K}^{2}
\end{aligned}
$$

The terms Term1,Term2,Term3 and Term4 are the closed form solution to the double intergrals which are obtained in [16].
For temperatures below the crust $\mathrm{z} \geq \mathrm{L}$ the mean temperature is obtained as

$$
T(z)=T(L)+\frac{Q_{B}}{\bar{K}}(z-L)
$$

Analytical expression for variance in temperature is derived. These expressions have been used to compute the subsurface thermal structure along with its error bounds both in the crust and below it. A Matlab based GUI is developed to compute and plot the temperature depth distribution along with its error bounds. The controlling thermal parameters such as the crustal thickness, radiogenic heat production, characteristic depth, surface temperature, basal heat flow, thermal conductivity, coefficient of variability in thermal conductivity and correlation length scale are all given on the screen. The graphical representation of the results is displayed on the screen which will be useful to the geo-scientific community dealing with subsurface thermal structure.

\section{NUMERICAL EXAMPLES AND DISCUSSION}

In understanding the thermal structure of the Earth there are several questions which need clear answers. Many of the controlling parameters that define the Earth's processes are not known with certainty. In such situations these controlling parameters can be defined in a stochastic framework and an average picture of the system behavior together with its error bounds can be quantified. In this study the radiogenic heat production, basal heat flow and mean thermal conductivity are considered to be a deterministic values whereas the thermal conductivity is assumed to be random parameter with a constant mean and a Gaussian correlation structure.

To demonstrate the use of the analytical solutions numerical values of the controlling input thermal parameters for a realistic Earth model for the Latur Earthquake region are given in [2] as

Surface temperature $\left(\mathrm{T}_{0}\right)-30\left({ }^{0} \mathrm{C}\right)$

Basal heat flow $\left(\mathrm{Q}_{\mathrm{B}}\right)-14\left(\mathrm{~mW} / \mathrm{m}^{2}\right)$

Radiogenic heat production (A) - $2.6\left(\mu \mathrm{W} / \mathrm{m}^{3}\right)$

Depth (L) - $37(\mathrm{~km})$

Mean thermal conductivity $\bar{K}-3.0\left(\mathrm{~mW} / \mathrm{m}^{2}\right)$

Coefficient of variability $\mathrm{C}_{\mathrm{k}-\mathrm{H}} 0.4$ and 0.5

Correlation length scale $1 / \rho-10 \mathrm{~km}, 20 \mathrm{~km}$

The lithospheric thickness is obtained as the depth of the intersection of the geotherm with a $1300{ }^{\circ} \mathrm{C}$ mantle adiabat. The numerical values of the above controlling parameters are given in the boxes and temperature depth distribution computed and plotted. For the above set of controlling thermal parameters and for coefficient of variability $\mathrm{C}_{\mathrm{k}} 0.4(40 \%$ error in the thermal conductivity) and a correlation length scale $1 / \rho$ i.e., $20 \mathrm{~km}$ the lithospheric thickness is computed and is seen to be around $272 \pm 11 \mathrm{~km}$. Figure 1 shows the plot of the GUI for the above mentioned thermal parameters. The errors are seen to exponentially increase up to the base of the crust i.e., up to $37 \mathrm{~km}$. Below the crust the errors are seen to increase linearly with depth. At the base of the lithosphere the temperatures are around $1300 \pm 51^{\circ} \mathrm{C}$. 
Next with an increase in the coefficient of variability i.e 0.5 around $50 \%$ error in the thermal conductivity values and for a correlation length scale $1 / \rho$ i.e., $20 \mathrm{~km}$ the lithospheric thickness computed is seen to be around $272 \pm 15 \mathrm{~km}$ (Figure 2). From the plot it is seen that at the base of the lithosphere the error bound on the temperature is around $1300 \pm 64^{\circ} \mathrm{C}$. Hence with an increase in the coefficient of variability in thermal conductivity there is an increase in the error bounds on the temperatures which result in an increase in the errors on the lithospheric thickness.

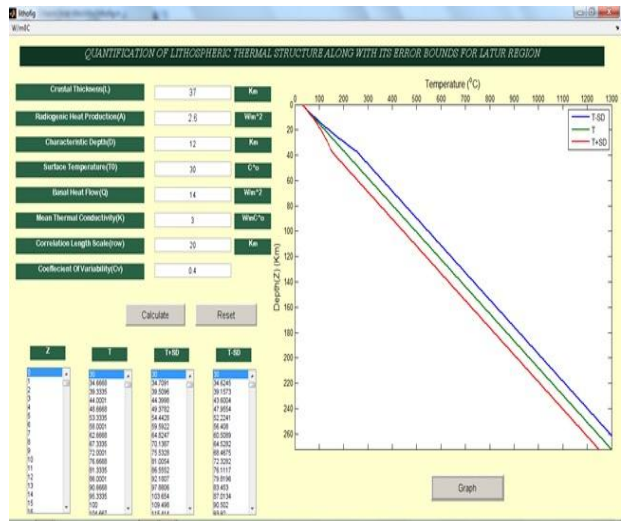

Figure 1: Plot of mean temperature \pm S.D for coefficient of variability in thermal conductivity 0.4 and a correlation length scale of $20 \mathrm{~km}$

Also to see the effect of a change in the correlation length scale on the errors the temperature depth distribution is computed with $40 \%$ error in the coefficient of variability in the thermal conductivity and for a correlation length scale of $10 \mathrm{~km}$. The lithospheric thickness computed is seen to be around $272 \pm 20 \mathrm{~km}$. The results are plotted in Figure 3. From the plot it is seen that at the base of the lithosphere the error bound on the temperature is around $1300 \pm 87^{\circ} \mathrm{C}$.

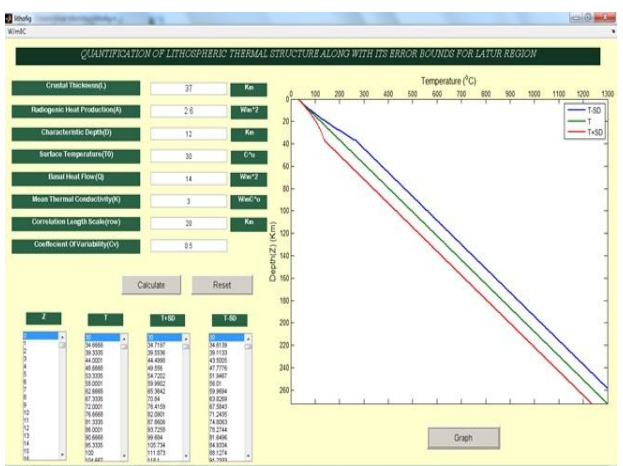

Figure 2: Plot of mean temperature \pm S.D for coefficient of variability in thermal conductivity 0.5 and a correlation length scale of $20 \mathrm{~km}$

For the Precambrian continental lithospheric the lithospheric thickness ranges from 140 to $350 \mathrm{~km}$ [17]. Latur region of India is characteristic of a Precambrian continental region. The results on lithospheric thickness obtained for the Indian region show that it is around the same Global average values of $272 \pm 20 \mathrm{~km}$.

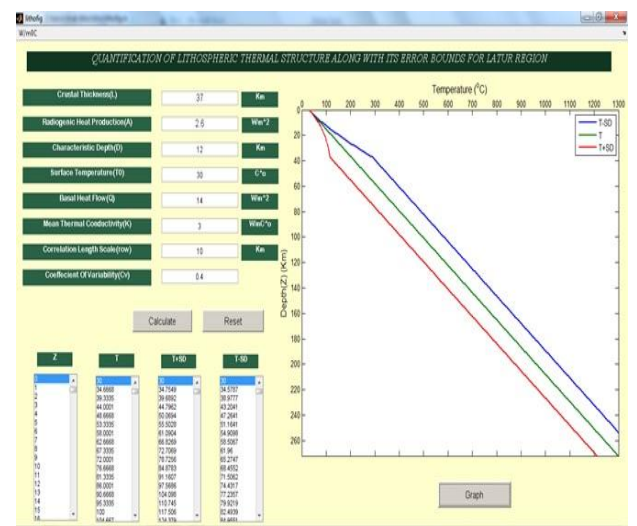

Figure 3: Plot of mean temperature \pm S.D for coefficient of variability in thermal conductivity 0.4 and a correlation length scale $10 \mathrm{~km}$

\section{CONCLUSIONS}

Thermal modeling plays an important role in understanding the state of the lithosphere. For modeling the conductive heat transfer in the crust the important controlling parameters are the radiogenic heat production and the thermal conductivity. In case of a stabilized continental crust the conductive heat transfer in steady state condition is a reasonably good approximation. A package is developed to compute and plot the error bounds on the subsurface temperatures due to errors in the thermal conductivity for a 1-D steady state conductive earth model for surface temperature and basal heat flow as its boundary conditions. A Graphical user interface has been developed in Matlab. Controlling input thermal parameters such as crustal thickness, radiogenic heat production, characteristic depth, surface temperature, surface heat flow, mean thermal conductivity, coefficient of variability in thermal conductivity and correlation length scale can be given directly on the screen and the plot of mean temperature along with its error bounds are displayed directly on the screen. Analytical expressions are derived below the crust also and when temperature reaches $1300^{\circ} \mathrm{C}$ the the computations stop and the corresponding depth gives the lithospheric thickness. This developed package is applied to Latur earthquake region in the India Shield and the lithospheric thickness along with the error bounds is quantified and is around $272 \pm 20 \mathrm{~km}$ which matches well with the documented results for any Precambrian shield.

\section{ACKNOWLEDGEMENTS}

The authors are thankful to the Director, National Geophysical Research Institute, Hyderabad for his kind permission to publish this work.

\section{REFERENCES}

[1] Vasseur G., Lucazeau. F. and Bayer, R.1985. The problem of heat flow density determination from inaccurate data, Tectonophysics, 121, 23-34.

[2] Srivastava, K. 2005. Modeling the variability of heat flow due to random thermal conductivity of the crust, Geophysical Journal International, 160 (2), 776-782.

[3] Vasseur, G., and Singh, R.N. 1986. Effect of random horizontal variation in radiogenic heat source distribution on its relationship with heat flow, Journal Geophysical Research, 91, 10397-10404.

[4] Nielson, S.B. 1987. Steady state heat flow in a random medium and linear heat flow heat production relationship, Geophysical Research Letters, 14, 318-321. 
[5] Srivastava, K., and Singh, R.N. 1998. A model for temperature variation in sedimentary basins due to random radiogenic heat sources, Geophysical Journal International, 135, 727-730.

[6] Royer J.J. and Danis, M. 1988. Steady state geothermal model of the crust and problems of boundary conditions: Application to a rift system, the southern Rhinegraben, Tectonophysics, 156, 239-255.

[7] Gallagher, K., Ramsdale, M., Lonergan. L., and Marrow, D. 1997. The role thermal conductivities measurements in modeling the thermal histories in sedimentary basins, Marine and Petroleum Geology, 14, 201-214.

[8] Jokinen. J. and Kukkonen. I.T. 1999b. Inverse simulation of lithospheric thermal regime using the Monte Carlo method, Tectonophysics, 306, 293-310.

[9] Jokinen. J. and Kukkonen. I.T. 1999a. Random Chapman, Stephen J. 2002. Matlab Programming for Engineers $\quad\left(2^{\text {nd }} \quad\right.$ Edition $), \quad$ Brooks/Cole. http://www.mathworks.com/discovery/matlab-gui.html.

[10] Witten, A. 2004. A Matlab based three dimensional viewer, Computers and Geosciences, 30, 693-703.

[11] Srivastava, K., Likhita Narain, Swaroopa Rani and V.P. Dimri. 2009. Quantification of crustal geotherms along with its error bounds for seismically active regions of India: A Matlab toolbox, Computers and Geosciences, $35,2009,2095-2099$.

[12] Pavan J., Ramana, D.V., and Chadha R.K 2012, A Matlab based GUI application in Hydroseismicity of the Koyna - Warna Region, India. International Journal of Computer Applications Volume 52(10), 38-43.

[13] Srivastava, K., Rohit Sharma, Bushra Fatima and R.N. Singh. 2006. Method for analytically obtaining closed form expressions for temperature depth distribution along with its error bounds, Patent No: 7, 130, 758, B2, Date of Patent, Oct 31, 2006.

[14] Srivastava, K. and Singh, R.N. 1999. A stochastic model to quantify the steady state crustal geotherms subject to uncertainty in thermal conductivity, Geophysical Journal International, 138, 895-899.

[15] Artemieva, IM and Mooney, WD. 2001. Thermal thickness and evolution of Precambrain lithosphere. Journal of Geophys. Res., 106, 16,387-16,414.

[16] Modeling of lithospheric thermal regime: Forward simulation applied in uncertainty analysis, Tectonophysics, 306, 277-292. 